\title{
Modeling and Generalized Predictive Control for Chilled Water in Central Air-conditioning System
}

\author{
Bai Yan ${ }^{1}$, Ren Qingchang ${ }^{2} \& \mathrm{Ma} \mathrm{Ke}^{3}$ \\ ${ }^{1}$ School of Science, Xi'an University of Architecture and Technology, Xi'an, China \\ ${ }^{2}$ School of Information and Control Engineering, Xi'an University of Architecture and Technology, Xi'an, China \\ ${ }^{3}$ School of Foreign Languages, Shaanxi Normal University, Xi'an, China \\ Correspondence: Bai Yan, School of Science, Xi'an University of Architecture and Technology, Xi'an, China. Tel: \\ 86-139-9191-9761. E-mail: yb_xauat@126.com
}

Received: July 7, 2013 Accepted: August 5, 2013 Online Published: August 14, 2013

doi:10.5539/cis.v6n4p25 URL: http://dx.doi.org/10.5539/cis.v6n4p25

\begin{abstract}
In this paper, the overall framework for chilled water system in heating, ventilation, and air-conditioning (HVAC) system was analyzed, then aiming at the chilled water system, the control loop in secondary pump frequency pressure difference was identified and the Generalized Prediction Control (GPC) algorithm was designed and deployed for the strategy of fixed pressure difference control. The GPC algorithm adopts multi-step prediction, rolling optimization and feedback correction method to suit a wide range of process. Simulation and experimental results demonstrate that the designed GPC algorithm has obvious advantages in effectiveness and superior performance with strong tracking and anti-jamming capability, compared with conventional manually tuned PID control algorithm.
\end{abstract}

Keywords: modeling, generalized predictive control, central air-conditioning system, chilled water

\section{Introduction}

HVAC systems require control of environmental variables such as pressure, temperature, humidity, etc. As one of the most important part in the central air conditioning system, chilled water circulation system distribute chilled water for air handling unit, and it's control effect will directly affect the operation of the whole system. At present, most of the controllers commissioned in HVAC systems are of the proportional-integral-derivative (PID) type because PID is simple yet sufficient for most HVAC application. Tuning a PID controller requires an accurate model of a process and an effective controller design rule. However, it is difficult to establish its precise mathematical model because of the system's nonlinearity, time variance, coupling and uncertainty. Meanwhile, the system control process is also in constant adjustment, which makes conventional PID control is difficult to achieve the desired control effect. Although many improved PID design methods are proposed, the Ziegler and Nichols (Z-N) methods are still adopted by many HVAC control engineers. The Z-N methods have shortcomings such as long testing time and limited control performance.

Clarke et al. (1987) propose GPC algorithm which adopts online recursive algorithm to estimate parameters in order to replace original model parameters on the basis of parametric model. As a result of multi-step prediction, rolling optimization and feedback correction, the useful information which reflects the dynamic behavior is fully utilized to enhance the robustness of delay and order change of the controlled object, so that the control performance is improved (Xi, 1993; Pawlowskia, Guzmána, Normey, \& Berenguela, 2012; Hu \& Jia, 2000).

Lim and Ling (1989) apply GPC algorithm to the control of the heat exchanger, and achieve good control effects. In central air-conditioning control field, some research institutions and scholars are dedicated to GPC algorithm, which is mainly used in simulation of ice melting process in ice storage air conditioning system, VAV (Variable Air Volume) terminal and room temperature control (Duan \& Ren, 2009; Yang, 2009).

In this paper, the GPC self-tuning controllers was designed and deployed on the VAV central air conditioning system experimental platform in Intelligent Building Institute in Xi'an University of Architecture and Technology. 


\section{The Overall Architecture of Chilled Water System in Central Air-Conditioning System}

This experimental research platform consists of water systems, air systems and terminal systems, and contains 2 water chillers, 2 air handling units (AHU), 6 terminals (VAVBOX), 3 fixed-frequency pumps, 1 variable frequency secondary pump and sensors, controllers and actuators, shown in Figure 1. The research of the chilled water system mainly focuses on the control loop in secondary pump frequency - pressure difference.

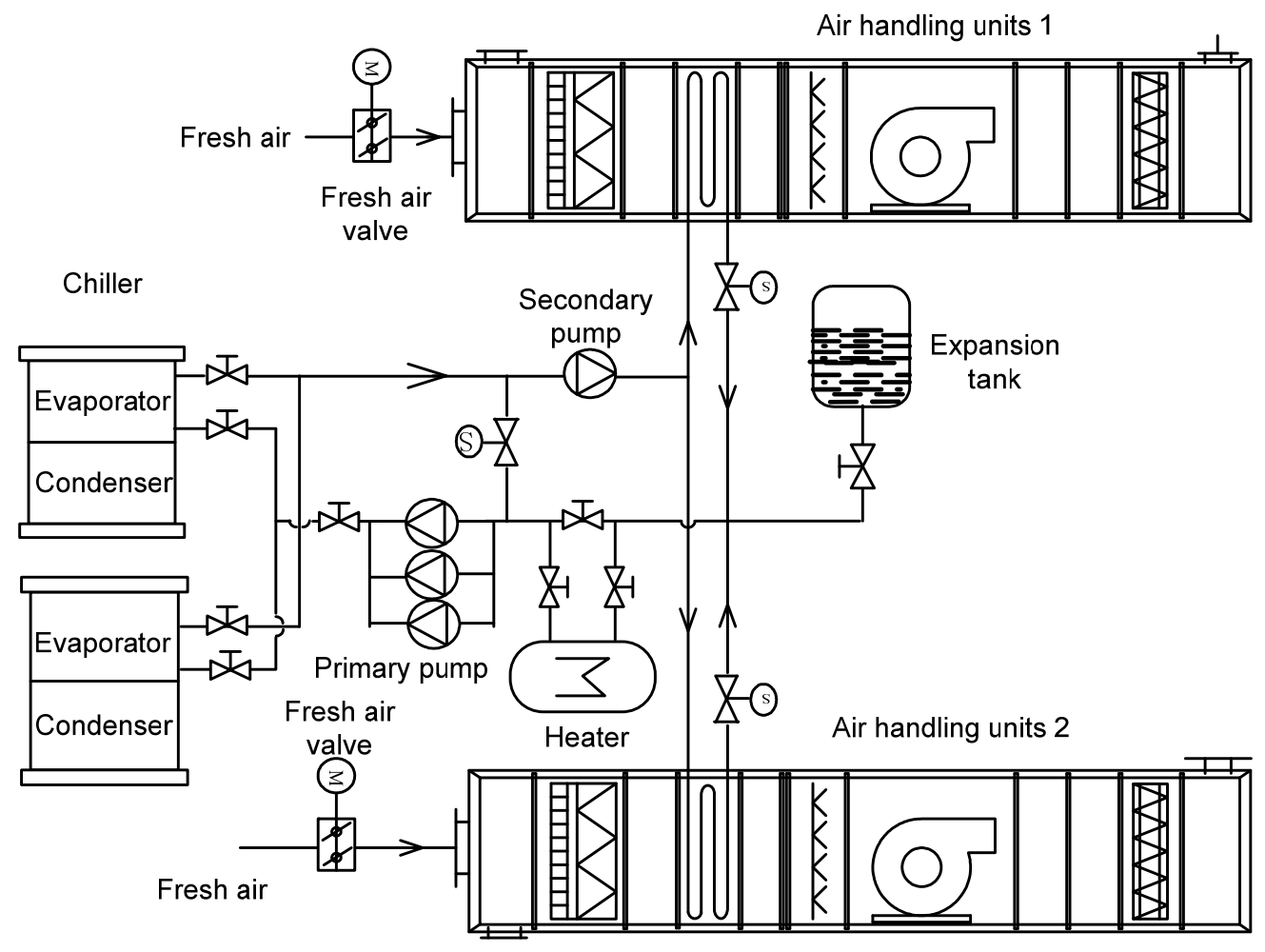

Figure 1. Architecture of chilled water system

\section{Chilled Water System Identification and Parameter Estimation}

\subsection{Basic Theory of System Identification}

According to L. A. Zadeh (1962), the system identification is a process of finding a model equal to the measurement system with input and output data from a given model class. Its rationale is shown in Figure 2.

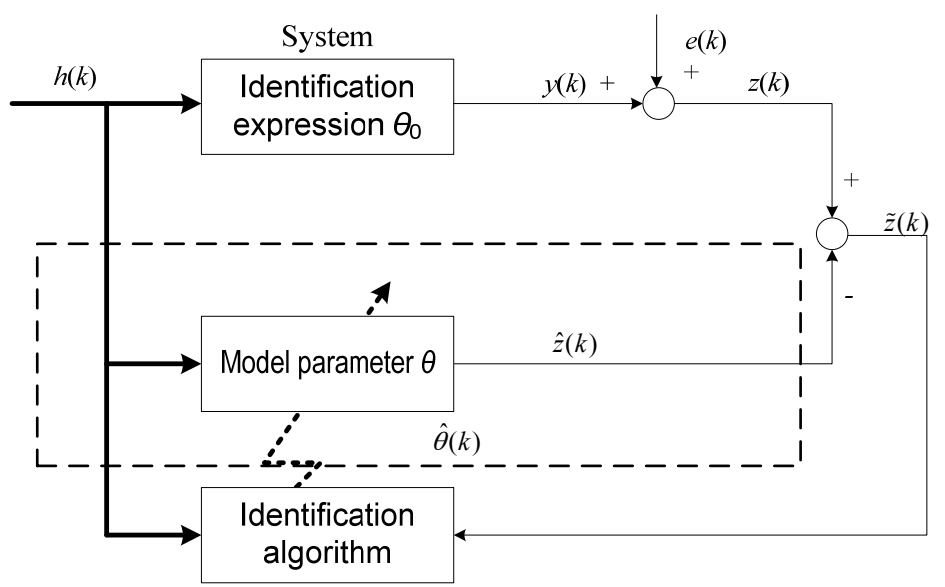

Figure 2. Rationale of system identification 
Usually, the successive approximation method is used to get the estimated value of model parameter $\theta$. At the time of $\mathrm{k}$, the model output value can be calculated out according to the estimated parameter value at the time of $k-1$, which is called predicted value.

$$
\hat{z}(k)=h^{T}(k) \hat{\theta}(k-1)
$$

And the prediction error $\tilde{z}(k)=z(k)-\hat{z}(k)$ can be calculated. The system output value (Figure 2)

$$
z(k)=h^{T}(k) \theta_{0}(k-1)+e(k)
$$

and the input of the identification expression $h(k)$ can be measured. The prediction error $\tilde{z}(k)$ is put into identification algorithm and the estimated parameter value $\hat{\theta}(k)$ in time $\mathrm{k}$ can be calculated under certain rule and accordingly, the model parameter can be updated. Thus, the iteration is done until the criterion function attains its minimum value. At this time, the model output $\hat{z}(k)$ is the best approximation to system output value $z(k)$ under such criterion to obtain the desired model (Bai \& Zhang, 2007; Kusiak \& Xu, 2012).

\subsection{Identification Algorithm}

In this paper, the least-squares algorithm was used for system identification. The model structure was determined firstly, and then the optimal parameters of the system model were determined under the structure. This method can be transformed into recursive form which gradually replaced the classical identification algorithm to be the most common method.

The system input and output relation can be expressed in the form of least squares.

$$
z(k)=h^{T}(k) \theta+e(k)
$$

In the Formula (3), $e(k)$ is the random noise and its mean is zero. When $K=1,2, \ldots, L$, the above formula can be expressed as matrix form

$$
Z_{L}=H_{L} \theta+e_{L}
$$

where $H_{L}=[h(1), h(2), \cdots, h(L)]^{T}, Z_{L}=[z(1), z(2), \cdots, z(L)]^{T}, e_{L}=[e(1), e(2), \cdots, e(L)]^{T}$. Taking criterion function,

$$
J(\theta)=\sum_{k=1}^{L}[e(k)]^{2}=\sum_{k=1}^{L}\left[z(k)-h^{T}(k) \theta\right]^{2}=\left(z_{L}-H_{L} \theta\right)^{T}\left(z_{L}-H_{L} \theta\right)
$$

and minimizing $J(\theta)$, the estimated parameter value of $\theta$ can be obtained to make the output of the model predicted accurately.

Let $\hat{\theta}_{L S}$ such that $\left.J(\theta)\right|_{\hat{\theta}_{L S}}=\min$, then

$$
\left.\frac{\partial J(\theta)}{\partial \theta}\right|_{\hat{\theta}_{L S}}=\frac{\partial}{\partial \theta}\left(z_{L}-H_{L} \theta\right)^{T}\left(z_{L}-H_{L} \theta\right)=0
$$

The formulas listed above were expanded and the two vectors differential equations were used, shown in Formulas (7) and (8).

$$
\begin{gathered}
\frac{\partial}{\partial x}\left(P^{T} x\right)=P^{T} \\
\frac{\partial}{\partial x}\left(x^{T} P x\right)=2 x^{T} P
\end{gathered}
$$

where $P$ is a symmetric matrix, the canonical equation can be drawn as

$$
\left(H_{L}^{T} H_{L}\right) \hat{\theta}_{L S}=H_{L}{ }^{T} z_{L}
$$

If $H_{L}{ }^{T} H_{L}$ is a regular matrix,

and

$$
\hat{\theta}_{L S}=\left(H_{L}^{T} H_{L}\right)^{-1} H_{L}^{T} z_{L}
$$

$$
\left.\frac{\partial^{2} J(\theta)}{\partial \theta^{2}}\right|_{\hat{\theta}_{L S}}=2 H_{L}^{T} H_{L}>0
$$

Therefore, the unique $\hat{\theta}_{L S}$ which satisfied Formula (11) made $\left.J(\theta)\right|_{\hat{\theta}_{L S}}=\min$. 
The calculation method for $\hat{\theta}_{L S}$ using minimization Formula (5) is called least squares method, and $\hat{\theta}_{L S}$ is called the least squares estimated value.

The least square model can be expressed as

$$
A\left(z^{-1}\right) z(k)=\frac{B\left(z^{-1}\right)}{F\left(z^{-1}\right)} u(k)+\frac{C\left(z^{-1}\right)}{D\left(z^{-1}\right)} v(k)
$$

where $A\left(z^{-1}\right), B\left(z^{-1}\right), C\left(z^{-1}\right), D\left(z^{-1}\right)$ and $F\left(z^{-1}\right)$ can be combined into different model classes.

$$
\begin{gathered}
A\left(z^{-1}\right)=1+a_{1} z^{-1}+a_{2} z^{-2}+\cdots+a_{n_{a}} z^{-n_{a}} \\
B\left(z^{-1}\right)=b_{1} z^{-1}+b_{2} z^{-2}+\cdots+b_{n_{a}} z^{-n_{b}} \\
F\left(z^{-1}\right)=1+f_{1} z^{-1}+f_{2} z^{-2}+\cdots+f_{n_{f}} z^{-n_{f}} \\
C\left(z^{-1}\right)=1+c_{1} z^{-1}+c_{2} z^{-2}+\cdots+c_{n_{c}} z^{-n_{c}} \\
D\left(z^{-1}\right)=1+d_{1} z^{-1}+d_{2} z^{-2}+\cdots+d_{n_{d}} z^{-n_{d}}
\end{gathered}
$$

$u(k)$ and $\mathrm{z}(k)$ are the input and output sequence of the model, $v(k)$ is unpredictable zero-mean white noise, $z^{-1}$ is the unit backward shift operator, $z^{-1} z(k)=z(k-1)$. The following models are usually used in identification:

- ARX model: $C\left(z^{-1}\right)=D\left(z^{-1}\right)=F\left(z^{-1}\right)=1$,

$$
A\left(z^{-1}\right) z(k)=B\left(z^{-1}\right) u(k)+v(k)
$$

- ARMAX model: $D\left(z^{-1}\right)=F\left(z^{-1}\right)=1$,

$$
A\left(z^{-1}\right) z(k)=B\left(z^{-1}\right) u(k)+C\left(z^{-1}\right) v(k)
$$

- DA(ARARX) model: $C\left(z^{-1}\right)=F\left(z^{-1}\right)=1$,

$$
A\left(z^{-1}\right) z(k)=B\left(z^{-1}\right) u(k)+\frac{1}{D\left(z^{-1}\right)} v(k)
$$

\subsection{Identification Results and Analysis}

In the experimental platform, the secondary pump is variable-speed pump, and the chilled water pressure control loop can be identified.

Step 1: The linear range of pressure changes are analyzed according to the characteristic curve of "frequency pressure" shown in Figure 3. The frequency range of the Secondary pump is $20-45 \mathrm{~Hz}$. It can be seen from Figure 3 that the linear interval of the second pump frequency and pressure is $20-40 \mathrm{~Hz}$.

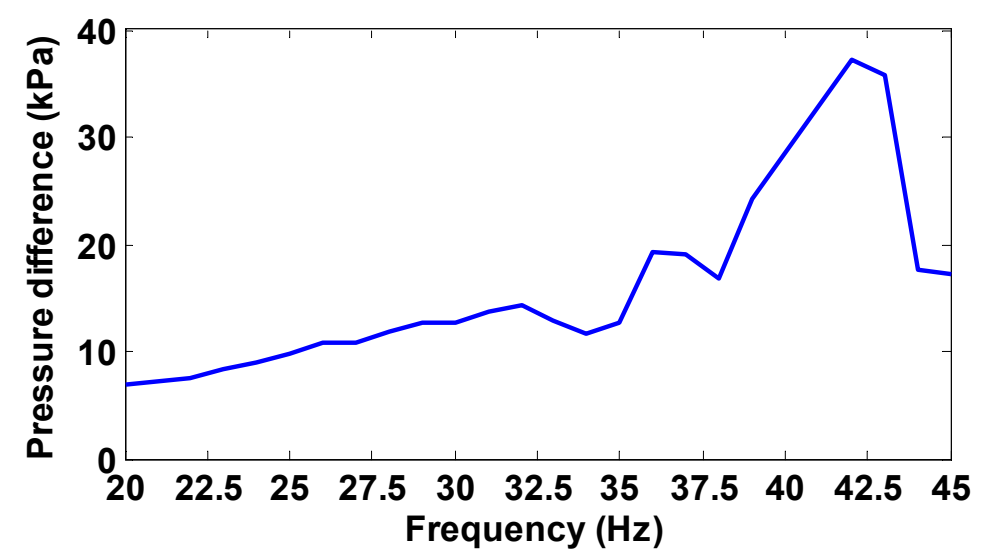

Figure 3. The characteristic curve of secondary pump frequency - pressure

Step 2: According to the step response of secondary pump frequency - pressure shown in Figure 4, the collected 
data show that the average delay time is 2.6 seconds and the average transient process time is 2 seconds.

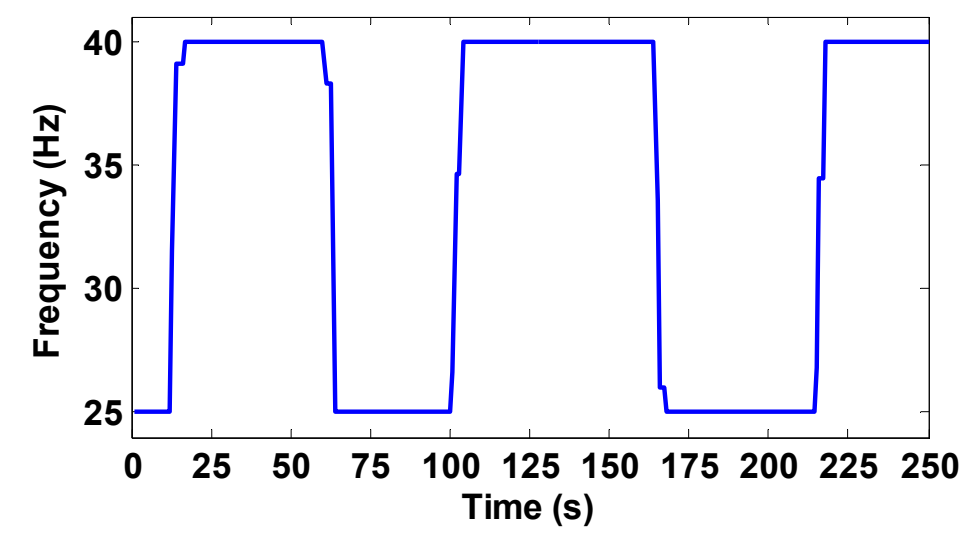

(a) Frequency of the secondary pump

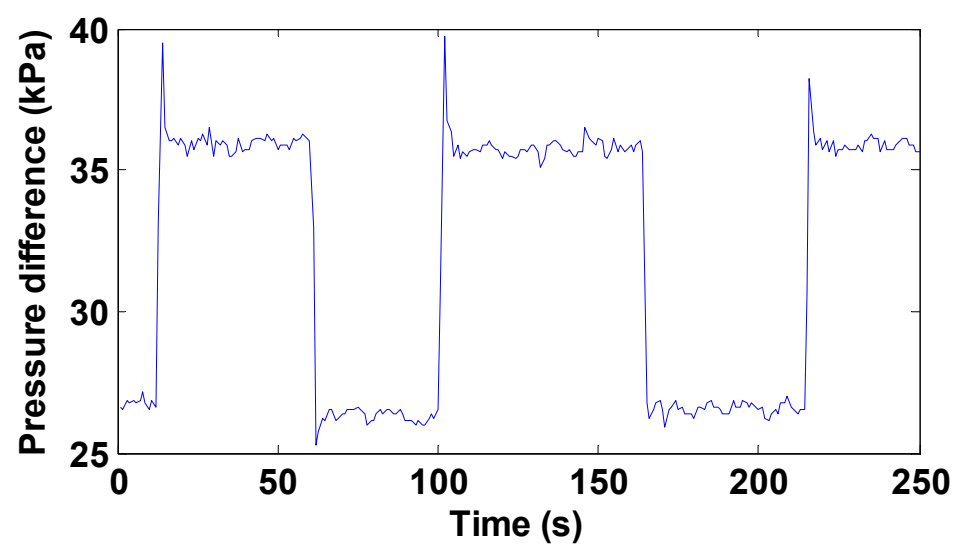

(b) Pressure of chilled water

Figure 4. The step response of secondary pump frequency - pressure

Step 3: The cutoff frequency is calculated by loading the secondary pump with the sinusoidal frequency signal. The amplitude of sinusoidal signal is 7.5 varying from 25 to $40 \mathrm{~Hz}$. The cutoff frequency $f_{\max }$ of the system is $0.18 \mathrm{~Hz}$ in the experiment.

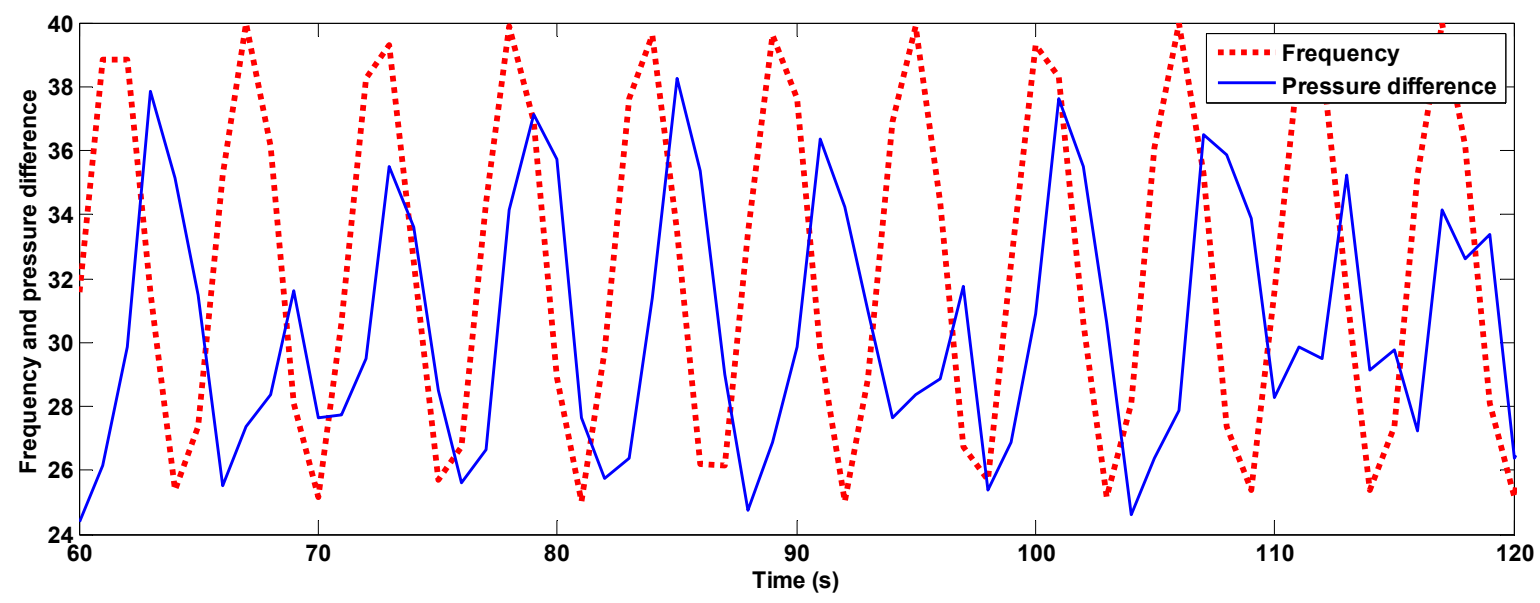

Figure 5. Pressure trend changes with frequency 
Step 4: Secondary pump frequency is loaded with M-sequence signal (Figure 6). M sequence sampling time $\Delta \mathrm{t}<=1 /\left(3^{*} f_{\max }\right)=2.08 \mathrm{~s}$, and let $\Delta \mathrm{t}=2 \mathrm{~s}$. The length of $\mathrm{M}$ sequence cycle $\mathrm{Np}>(\mathrm{Ts}+\Delta \mathrm{t}) / \Delta \mathrm{t}$, and as a result $\mathrm{Np}>3$. $\left(2^{\mathrm{n}}-1\right)>\mathrm{Np}=3$, let $\mathrm{n}=3$. M sequence cycle $\mathrm{T} \mathrm{p}=(\mathrm{Np}-1) * \Delta \mathrm{t}=\left(2^{3}-1\right) * 2=7 * 2=14 \mathrm{~s}$.
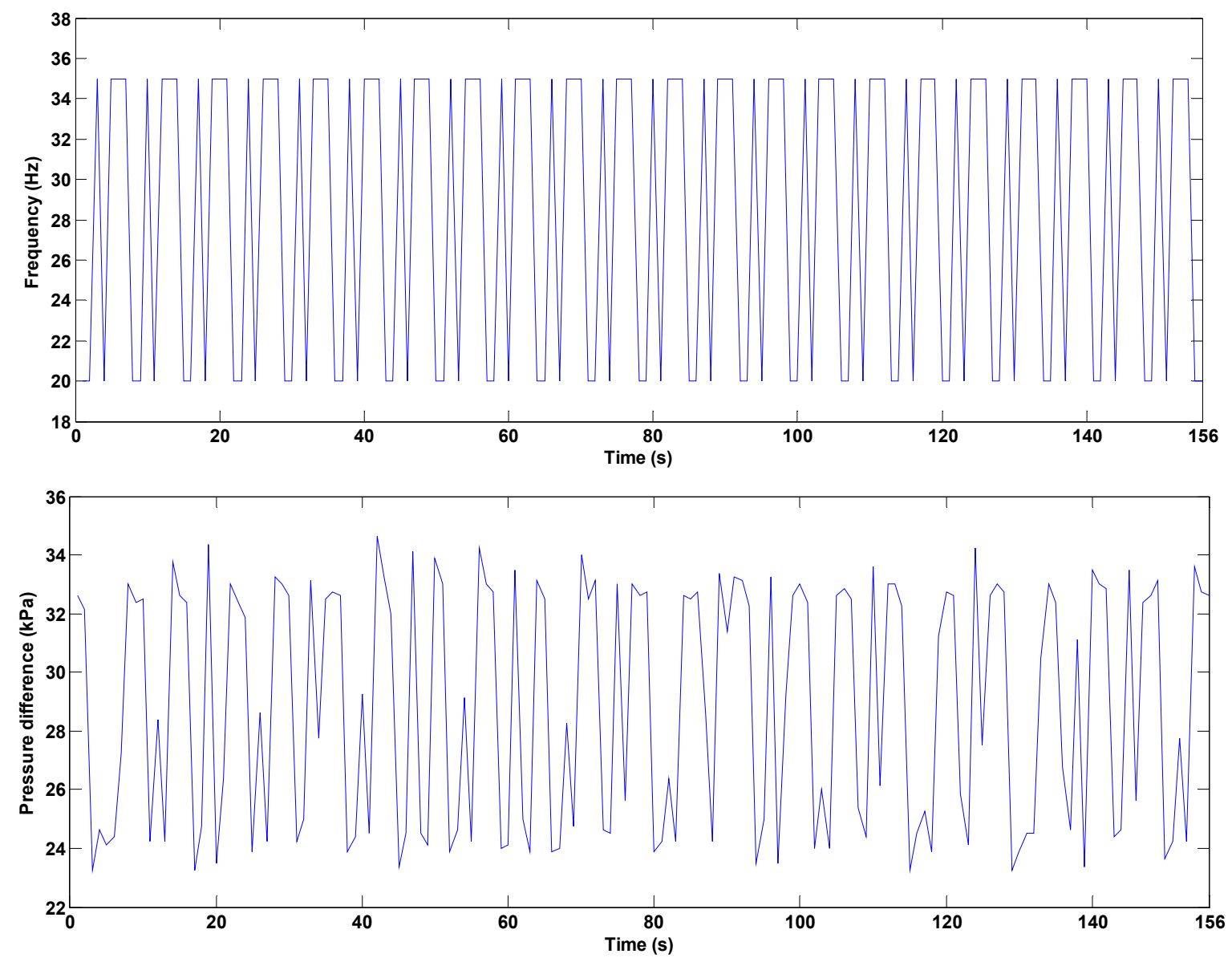

Figure 6. Input and output data of control circuit in secondry pump frequency - pressure

After a least squares identification, the ARX441 model can be described as Formula (16).

$$
G\left(z^{-1}\right)=\frac{0.07934 z^{-1}+0.3503 z^{-2}+0.5452 z^{-3}+0.1322 z^{-4}}{1-0.2248 z^{-1}-0.04842 z^{-2}+0.07979 z^{-3}-0.1995 z^{-4}}
$$

\section{Design and Simulation of GPC Algorithm}

\subsection{Generalized Predictive Control}

An $\mathrm{n}$ input, $\mathrm{n}$ output multi-variable is represented by the following controlled autoregressive and integrated moving average (CARIMA) model (Xu \& Li, 2007):

$$
A\left(z^{-1}\right) y(t)=B\left(z^{-1}\right) u(t-1)+C\left(z^{-1}\right) \xi(t) / \Delta
$$

where $A\left(z^{-1}\right), B\left(z^{-1}\right), C\left(z^{-1}\right)$ are the polynomials of retrusive operator $z^{-1}$.

$$
A\left(z^{-1}\right)=I_{n \times n}+A_{1} z^{-1}+\cdots+A_{n_{A}} z^{-n_{A}}, \quad B\left(z^{-1}\right)=B_{0}+B_{1} z^{-1}+\cdots+B_{n_{B}} z^{-n_{B}}, C\left(z^{-1}\right)=I_{n \times n}+C_{1} z^{-1}+\cdots+C_{n_{C}} z^{-n_{C}}, \quad \Delta=\operatorname{diag}\left\{1-z^{-1}\right\},
$$

where $y(t), u(t)$ and $\xi(t)$ are $n \times 1$ vector of output, input and noises respectively, and $\xi(t)$ is irrelevant white noise sequence. For simplicity, the matrix $C\left(z^{-1}\right)=I_{n \times n}$. 
The multi-stage cost function has the following form:

$$
J=E\left\{\sum_{j=N_{1}}^{N_{2}}\left[\hat{y}(k+j)-y_{r}(k+j)\right]^{2}+\sum_{j=1}^{N_{u}} \Lambda(j)[\Delta u(k+j-1)]^{2}\right\}
$$

where $\mathrm{E}$ is the mathematical expectation, $\Lambda(j)=\lambda I$ is the control weighting coefficient matrix, $\Delta u(k+j-1)$ is the control increment matrix, $\hat{y}(k+j)$ is a $k$-step prediction output at the time of $j$, and $y_{r}(k+j)$ is the reference trajectory determined by the following formula:

$$
\begin{aligned}
& y_{r}(k)=y(k), \\
& y_{r}(k+j)=\Gamma y_{r}(k+j-1)+(1-\Gamma) y_{s}(k)
\end{aligned}
$$

where $y_{\mathrm{s}}(k)$ is the set value, $N_{1}, N_{2}, N_{3}$ are minimum predicted time domain, maximum predicted time domain and control time domain respectively. Let $\mathrm{N}=N_{2}-N_{1}=N_{u}$. Softness factor matrix $\Gamma=\alpha I$, where $\alpha \in[0,1]$.

Establish Diophantine equation:

$$
\left\{\begin{array}{c}
I_{n \times n}=E_{j}\left(z^{-1}\right) A\left(z^{-1}\right) \Delta+z^{-j} F_{j}\left(z^{-1}\right) \\
E_{j}\left(z^{-1}\right) B\left(z^{-1}\right)=G_{j}\left(z^{-1}\right)+z^{-j} H_{j}\left(z^{-1}\right)
\end{array}\right.
$$

where $E_{j}, F_{j}, G_{j}, H_{j}$ are unique polynomial matrices of order $j$-1. Therefore, j-step optimal predictive control is

$$
\hat{y}(k+j)=G_{j} \Delta u(k+j-1)+F_{j} y(k)+H_{j} \Delta u(k-1)
$$

Through minimizing the objective cost function, the future optimal control variable is

$$
U=\left(G^{T} G+\Lambda\right)^{-1} G^{T}\left[y_{r}(k)-F y(k)-H \Delta u(k-1)\right]
$$

From the above, define

$$
G=\left[\begin{array}{cccccc}
G_{0} & 0 & \cdots & 0 & \cdots & 0 \\
G_{1} & G_{0} & \cdots & 0 & \cdots & 0 \\
\vdots & \vdots & \ddots & \vdots & \vdots & \vdots \\
G_{j-1} & G_{j-2} & \cdots & G_{0} & \vdots & 0 \\
\vdots & \vdots & \vdots & \vdots & \ddots & \vdots \\
G_{N-1} & G_{N-2} & \cdots & \cdots & \cdots & G_{0}
\end{array}\right]_{N \times N}
$$

\subsection{Simulation of the Control Algorithm}

The supply and return water pressure is the controlled object in chilled water system. The generalized predictive control principle used in this paper is shown in Figure 7. In the figure, $w(k)$ is the set value of differential pressure, $y_{r}(k+i)$ is reference trajectory, $y_{e}(k+i)$ is corrected output, $u(k)$ is control input, $d(k)$ is random disturbance, $y_{m}(k+i)$ is the calculated value of prediction models, $y(k)$ is the current output value of the pressure difference.

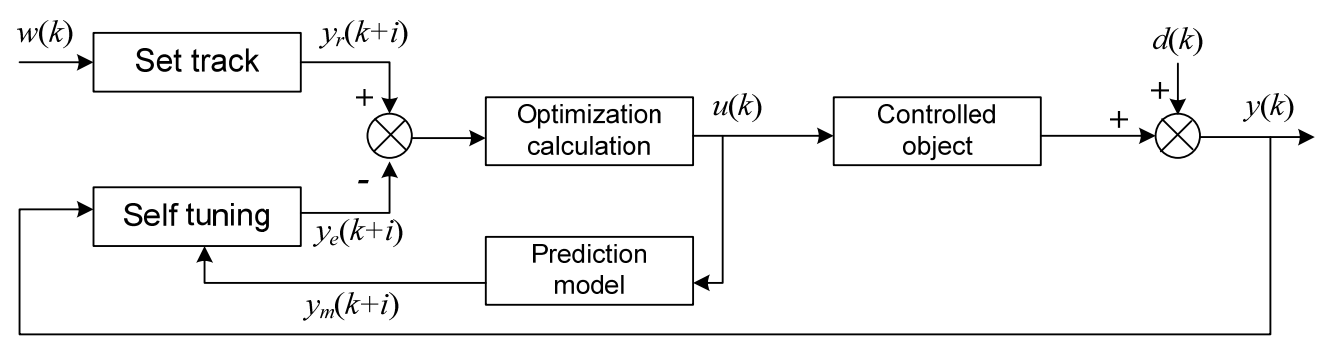

Figure 7. Schematics of generalized predictive control

The simulation control results of ZN-PID and GPC algorithm are shown in Figure 8, with differential pressure setting of $100 \mathrm{kPa}$. 


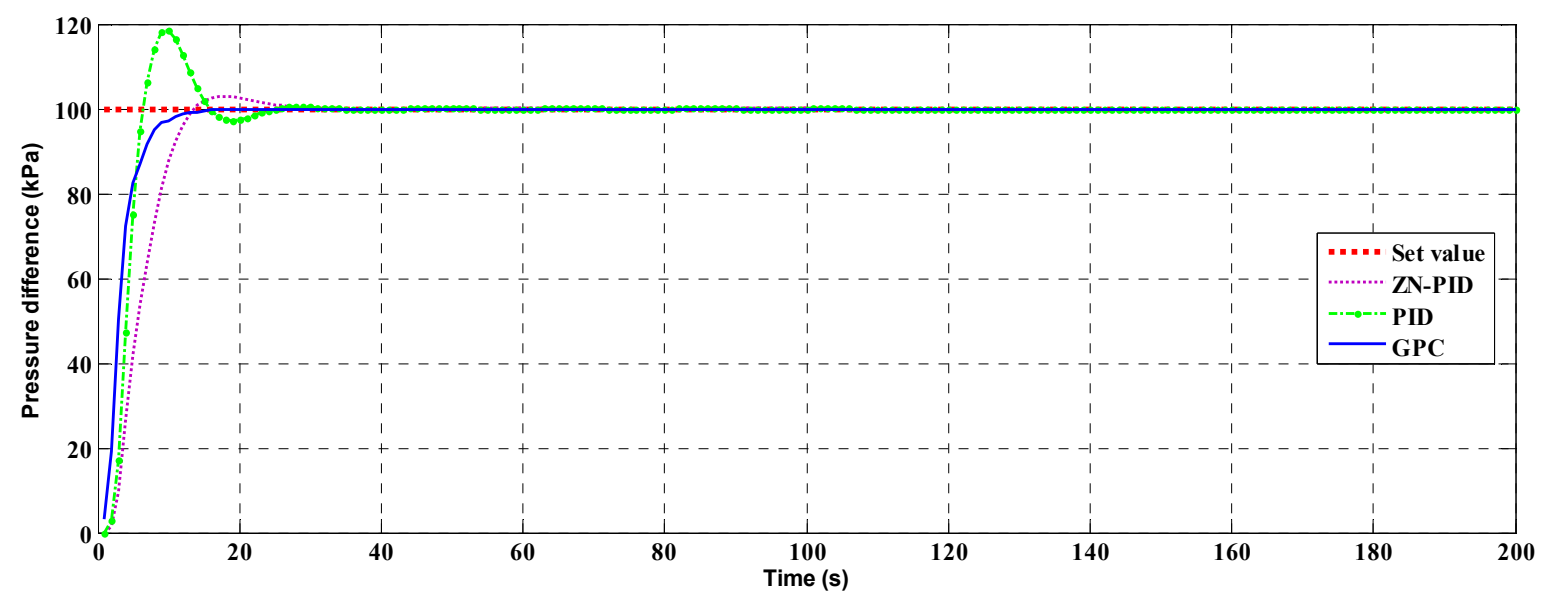

Figure 8. Simulation results comparison chart

In this control process, time domain performance comparison is as shown in Table 1.

Table 1. Time domain performance comparison of ZN-PID and GPC

\begin{tabular}{cccc}
\hline & PID & ZN-PID & GPC \\
\hline Overshoot $/ \%$ & 18.6 & 3 & 0 \\
Rise time $/ \mathrm{s}$ & 7 & 14 & 14 \\
Peak time /s & 10 & 18 & 19 \\
Settling time $/ \mathrm{s}$ & 6 & 12 & 8 \\
\hline
\end{tabular}

As can be seen from the time domain, the overshoot of PID algorithm, ZN-PID algorithm and the GPC algorithm were $18.6 \%, 3 \%$ and $0 \%$, the rise time are $7 \mathrm{~s}, 14 \mathrm{~s}$ and $14 \mathrm{~s}$, peak time are $10 \mathrm{~s}, 18 \mathrm{~s}$ and $19 \mathrm{~s}$, settling time are $6 \mathrm{~s}$, $12 \mathrm{~s}$ and $8 \mathrm{~s}$ respectively. Therefore, GPC algorithm achieves the best performance.

\section{Experimental Results and Analysis}

PID and GPC algorithm were adopted respectively for the control loop of the secondary pump constant pressure difference in chilled water system, and the control results were compared and analysed.

\subsection{The Response of a Setting Value}

Pressure difference setpoint changes from $76 \mathrm{kPa}$ to $90 \mathrm{kPa}$, and chilled water pressure difference using ZN-PID control algorithm is shown in Figure 9.

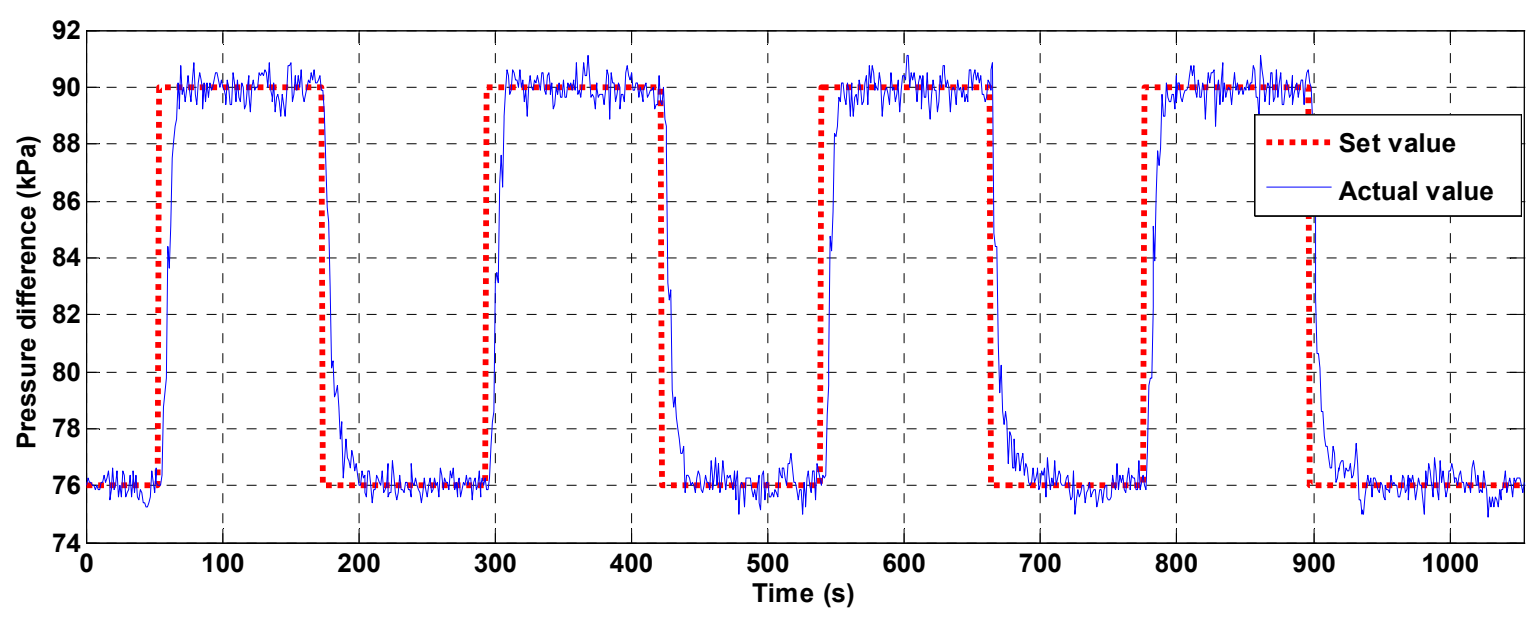

Figure 9. Step control for constant pressure difference with ZN-PID algorithm 


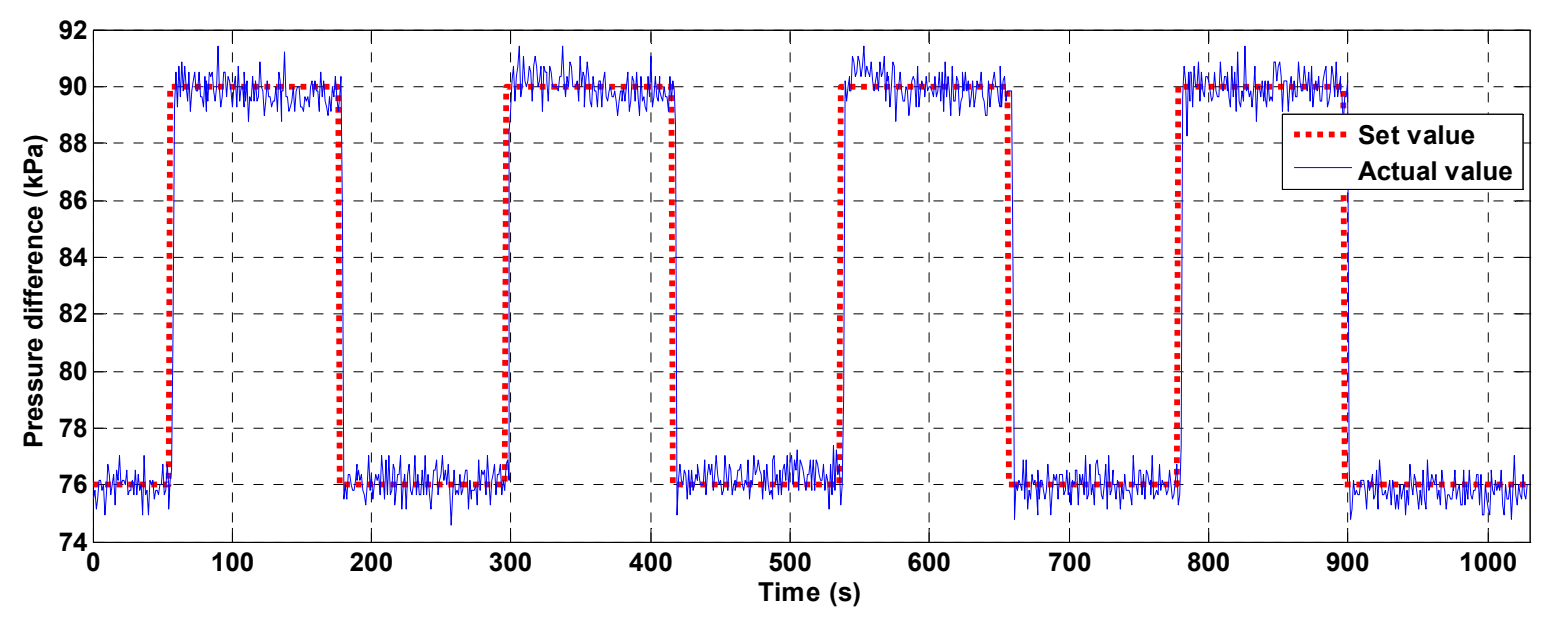

Figure 10. Step control for constant pressure difference with GPC algorithm

The above experimental results show that PID and GPC algorithm has good following performance to step control. Comparing the control results from Figure 9 and Figure 10, while overshoot of the PID algorithm is $0.83 \%$, settling time of rising edge and fall edge are $14 \mathrm{~s}$ and $29 \mathrm{~s}$ respectively, overshoot of the GPC algorithm is $1.56 \%$, settling time of rising edge and fall edge are both $10 \mathrm{~s}$.

\subsection{Disturbance Response}

\subsubsection{Disturbance Response Based on NN-PID Algorithm}

With the pressure difference setpoint of $90 \mathrm{kPa}$, the load changes of the two AHUs lead to the changes of the valve opening, and thereby causing the chilled water flow changes. System adjusts the secondary pump frequency to maintain the pressure difference setpoint based on PID control algorithm. Control results are shown in Figure 11. And chilled water valve opening and chilled water flow are shown in Figure 12 and Figure 13 respectively.

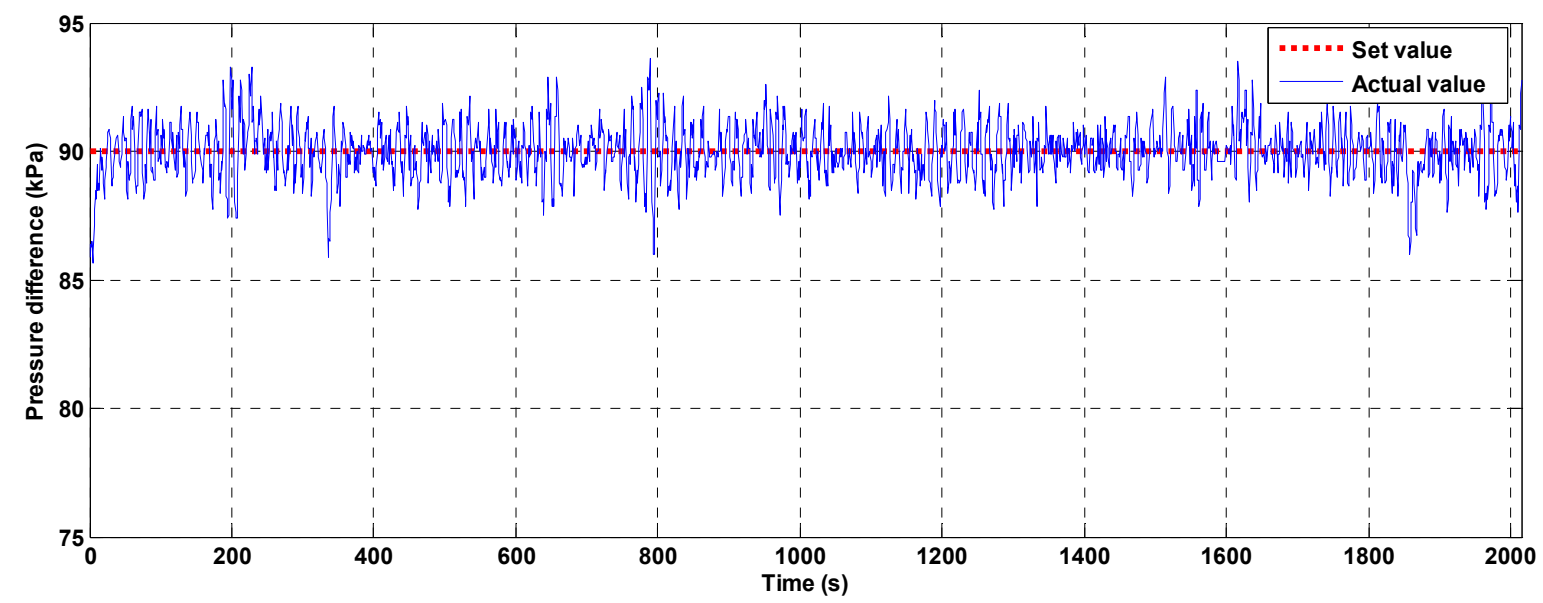

Figure 11. PID algorithm based constant pressure difference control 


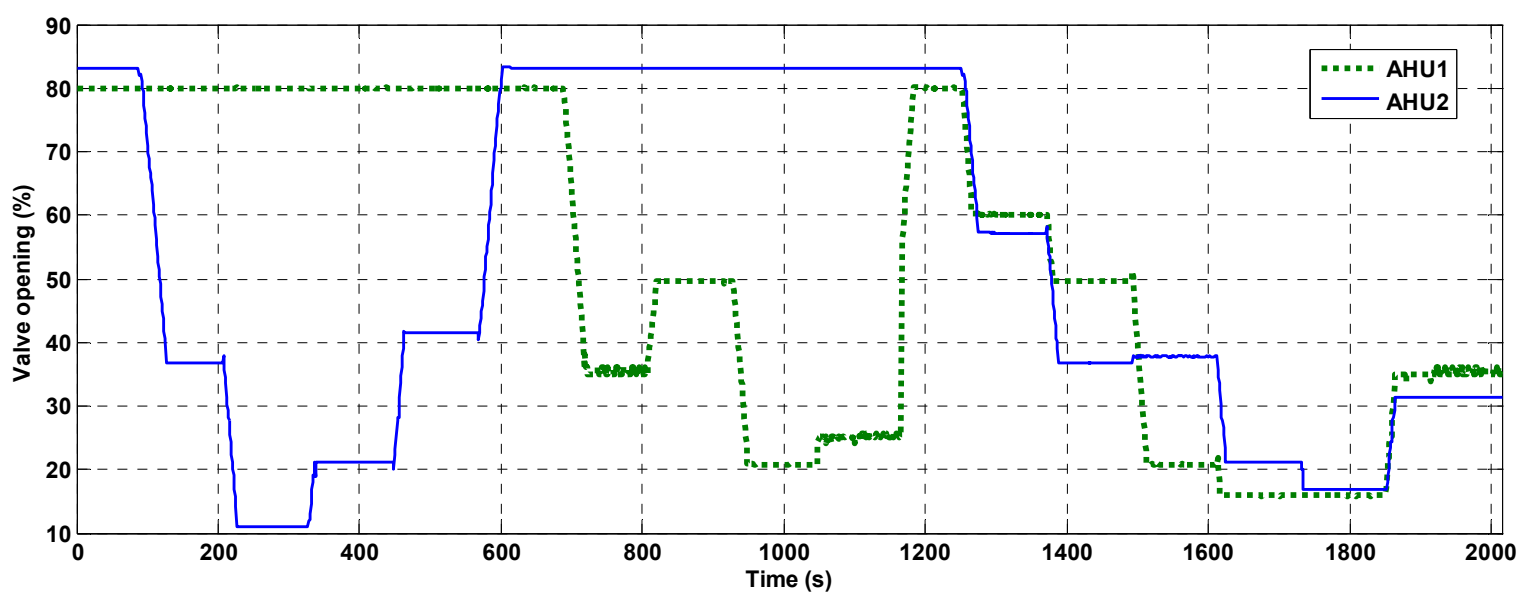

Figure 12. Chilled water valve opening change of AHU1 and AHU2

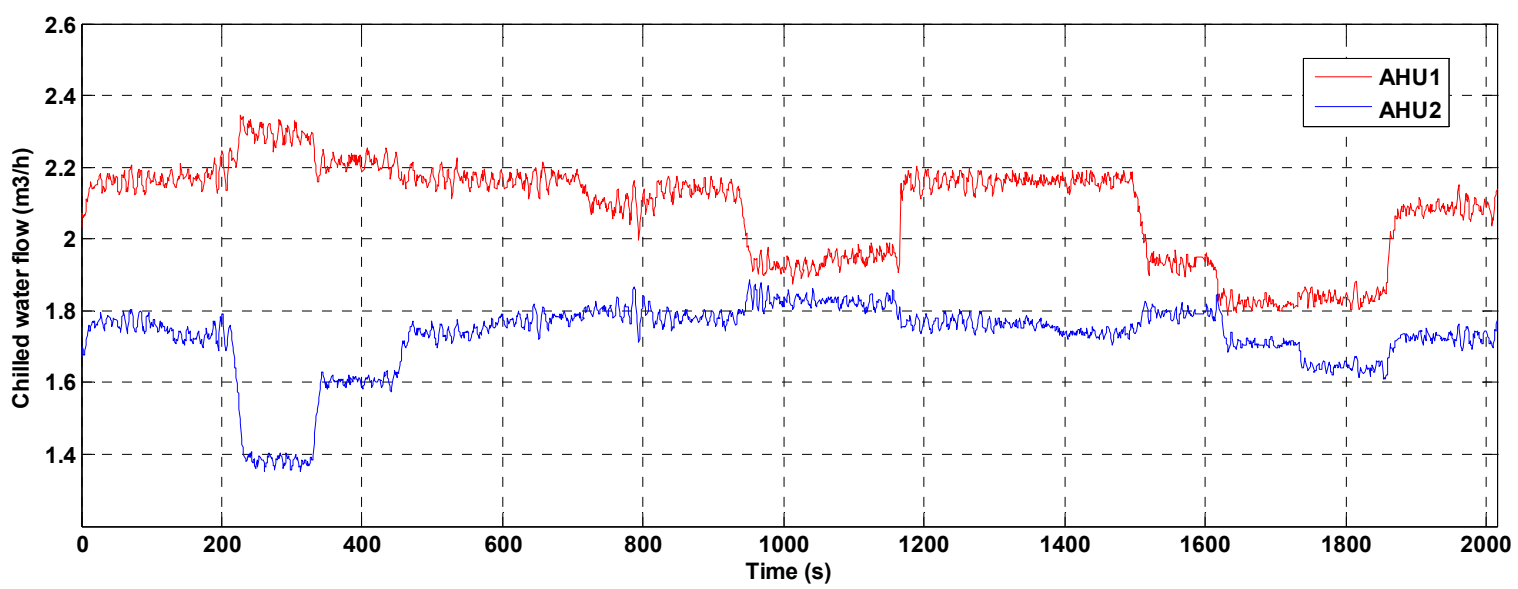

Figure 13. Chilled water flow change of AHU1 and AHU2

\subsubsection{Disturbance Response Based on GPC Algorithm}

With the pressure difference setpoint of $86 \mathrm{kPa}$, the load changes of the two AHUs lead to the changes of the valve opening, and thereby causing the chilled water flow changes. System adjusts the secondary pump frequency to maintain the pressure difference setpoint based on GPC control algorithm. Control results are shown in Figure 14 and chilled water valve opening and chilled water flow, shown in Figure 15 and Figure 16 respectively.

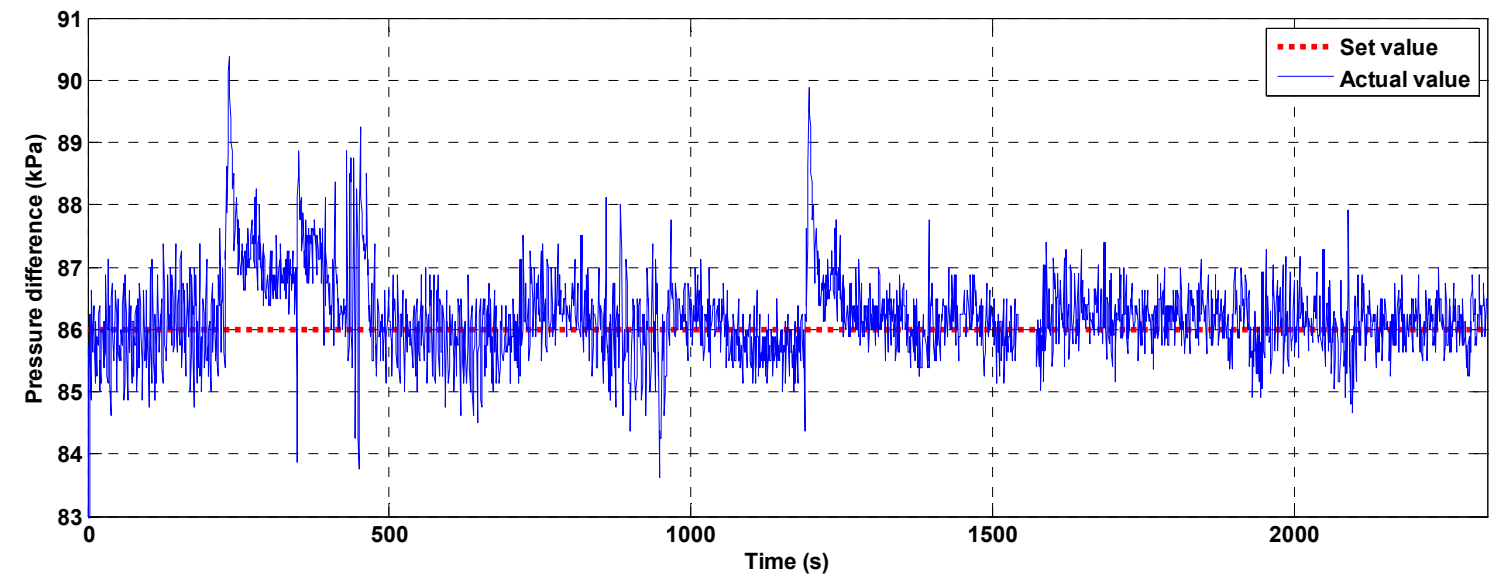

Figure 14. GPC algorithm based constant pressure difference control 


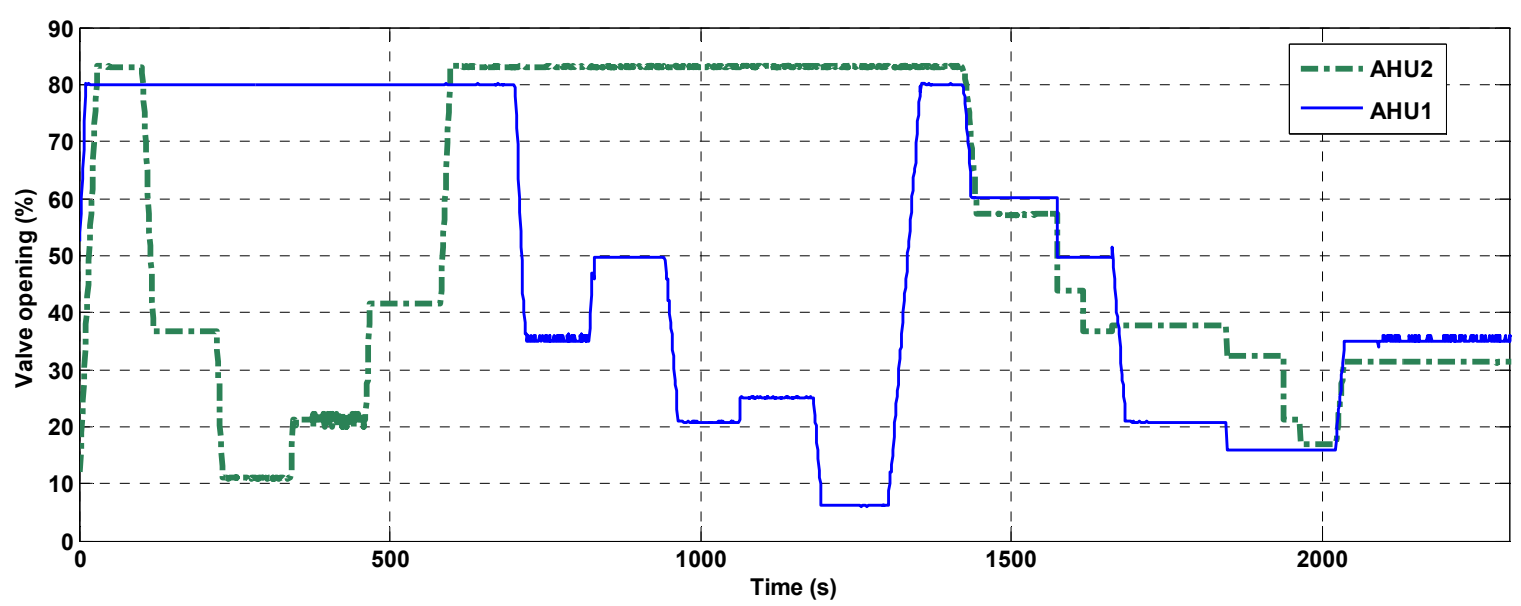

Figure 15. Chilled water valve opening change of AHU1 and AHU2

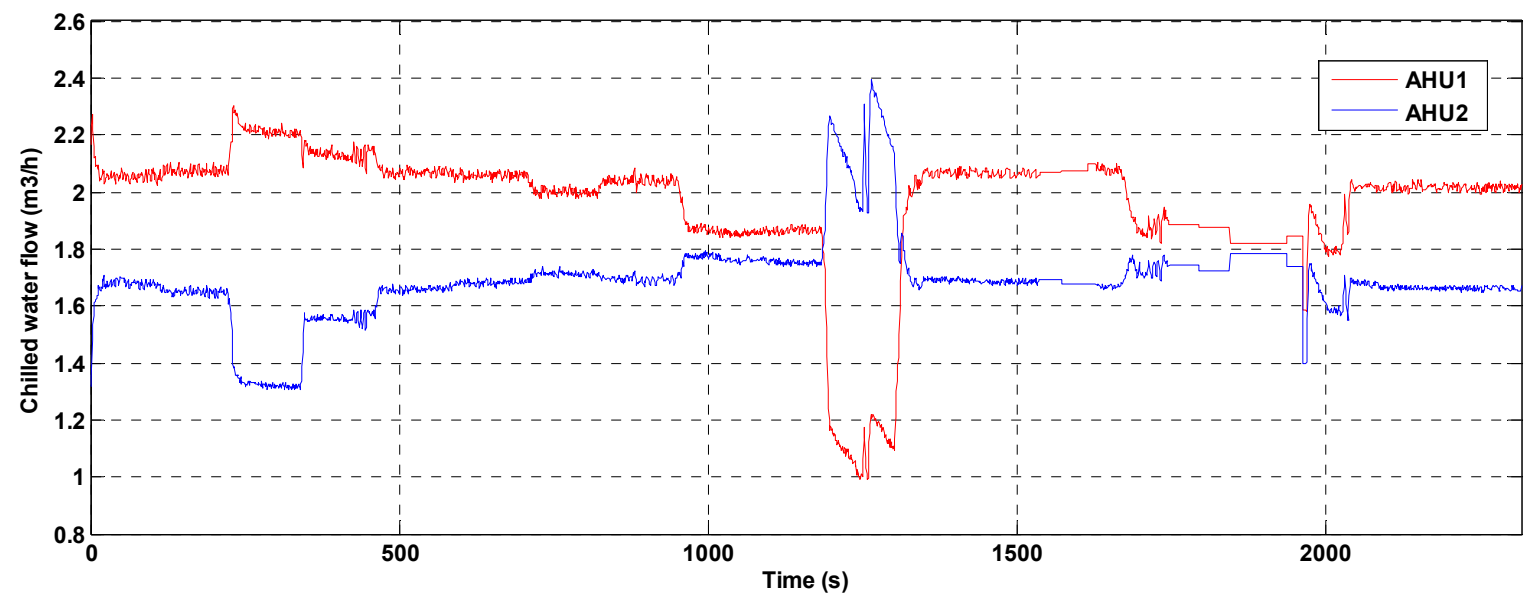

Figure 16. Chilled water flow change of AHU1 and AHU2

Figure 11 and Figure 14 show that the PID algorithm is more stable, however GPC algorithm can quickly reject the disturbance caused by valve opening and reach the set value quickly.

\section{Conclusions}

In this paper, the GPC algorithm was designed and deployed on the constant pressure difference control strategy for chilled water based on the modeling of the secondary pump frequency - pressure control loop. Simulation and experimental results show that the designed GPC algorithm has strong anti-jamming capability and tracking performance, and can be used for central air conditioning water system.

\section{Acknowledgements}

This work was financially supported by the Foundation of Educational Commission of Shaanxi Province of China (No.11JK0906), and the ministry of housing and urban-rural development of China (2012-K1-35).

\section{References}

Bai, J. B., \& Zhang, X. S. (2007). Online identification algorithm for air conditioning systems. $H V \& A C, 37(5)$, $18-23$.

Clarke, D. W. (1987). Generalized Predictive Control—Part II Extensions and interpretations. Automatica, 23(2), 149-160. http://dx.doi.org/10.1016/0005-1098(87)90088-4

Duan, F. Y., \& Ren, Q. C. (2009). Application of GPC Algorithm in Ice-storage Air-conditioning Control System. Equipment Manufactring Technology, 6, 79-80.

Hu, Y. H., \& Jia, X. L. (2000). Summarization of generalized predictive control. Information and Control, 29(3), 
248-256.

Kusiak, A., \& Xu, G. L. (2012). Modeling and optimization of HVAC systems using a dynamic neural network. Energy, 42, 241-250. http://dx.doi.org/10.1016/j.energy.2012.03.063

Lim, K. W., \& Ling, K. V. (1989). Generalized predictive control of a heat exchanger. IEEE Control Systems M agazine, 9(5), 9-12. http://dx.doi.org/10.1109/37.41437

Pawlowskia, A., Guzmán, J. L., Normey-Rico, J. E., \& Berenguel, M. (2012). Improving feedforward disturbance compensation capabilities in Generalized Predictive Control. Journal of Process Control, 22(3), 527-539. http://dx.doi.org/10.1016/j.jprocont.2012.01.010

Xi, Y. G. (1993). Prediction control. Bei Jing, BJ: National Defence Industry Press.

Xu, M., \& Li, S. Y. (2007). Practical generalized predictive control with decentralized identification approach to HVAC systems. Energy Conversion and Management, 48, 292-299. http://dx.doi.org/10.1016/j.enconman.2006.04.012

Yang, H. X. (2009). Research of VAV terminal simulation and control based on generalized predictive self-tuning controller. Bei Jing, BJ: Beijing University of Technology.

\section{Copyrights}

Copyright for this article is retained by the author(s), with first publication rights granted to the journal.

This is an open-access article distributed under the terms and conditions of the Creative Commons Attribution license (http://creativecommons.org/licenses/by/3.0/). 